\title{
Nutritional Management of Overweight and Obesity in Dogs and Cats
}

\author{
Sorana MATEI, Andrei SZAKACS, Mara CĂPÎLNEAN, Adrian MACRI* \\ University of Agricultural Sciences and Veterinary Medicine Cluj-Napoca, Faculty of Veterinary Medicine, \\ 3-5 Manastur Street, Cluj-Napoca, Romania \\ * corresponding author: adimacri@yahoo.com \\ Bulletin UASVM Veterinary Medicine 74(1) / 2017, \\ Print ISSN 1843-5270; Electronic ISSN 1843-5378 \\ DOI:10.15835/buasvmcn-vm: 12609
}

\begin{abstract}
Some of the most common nutritional disorders are overweight and obesity, a proportion of approximately $59 \%$ of dogs and cats being affected. A permanent challenge for vets is weight management, including the prevention and treatment of overweight and obesity.

Corporeal score and body-weight loss in dogs and cats have been monitored by feeding various diets. The study was conducted on a total of 10 animals ( 6 dogs and 4 cats), monitoring the effect of three types of food for dogs and two types for cats suffering from overweight and obesity. Cooked food, dry food diet and premium dry food were investigated. We determined the quality and gross chemical composition of food and we measured corporeal score, weekly weight loss percentage and the number of calories consumed daily. We also appreciated the quality of life and activity level of the animals at the beginning and at the end of the trial. Nutritional management of investigated diets for overweight and obesity in dogs and cats revealed that through the smallest caloric restriction, dry food diet presented the highest efficiency, dogs and cats loosing weight steadily without losing muscle mass. Although the satiety effect occurs when the animals reach their ideal weight, the Rebound effect was not present.
\end{abstract}

Keywords: diet, management, obesity, overweight

\section{INTRODUCTION}

Obesity is the exaggeration of good maintenance condition characterized by the accumulation of fat at the level of subcutaneous and perivisceral tissue, this modification affecting the health state of the animal. The problem of obesity and overweight is increasing, a study done in 2006 showed that $24-44 \%$ of the population of dogs and cats is suffering from this medical problem (Pibot et al., 2010). It is considered that animals which have $10-20 \%$ more than the ideal body weight are suffering from obesity (Burns and Towell, 2011; Burns, 2013). Now it is no longer applied, being replaced by a body index test. The ideal situation is if we know the weight of the animal before fattening, this being impossible to dogs who suffered from obesity at an early age (Fascetti and Delaney, 2012).

Small breeds are more prone to obesity, while in the large ones was demonstrated that obesity is caused by musculoskeletal problems (Fascetti and Delaney, 2012).

Most of the times, the owners of obese dogs and cats are also obese themselves, 54\% believing that any gesture of dog relates to food. They tend to give an increased number of rewards and food scraps (Brooks et al., 2014; German et al., 2011; German et al.,2012). Approximately 59\% of dogs 
and cats is suffering of overweight and obesity, making it one of the most common disease in the current practice (Brooks et al., 2014).

Obesity is associated to cardiovascular musculoskeletal diseases, as well to respiratory, and kidney disease, skin disorders, increasing the risk of endocrine and metabolic diseases (Linder and Parker, 2016).

The aim of this study was to monitoring the corporeal score and body-weight loss in dogs and cats by feeding different types of diets.

\section{MATERIALS AND METHODS}

During this study, it was followed the effect of the administration of three types of dog food and two types of cat food suffering from overweight, respectively from obesity. There was determined the gross chemical composition and the quality of food administered by following the corporal score, the percentage of weekly weakening, the number of calories consumed daily, and we appreciated the quality of life and the activity level of the animal.

The study was conducted in 2016 on a total number of 10 animals (6 dogs and 4 cats). In order to achieve the Protocol there were formed 3 groups of 2 dogs, and 2 lots of 2 cats which have been given a certain type of food, being monitored throughout the whole study period of 24 weeks.

The types of food studied are of premium type, veterinary diet in form of granules and processed food. Cooked food was given to 2 dogs, and the quantity has been adjusted in time. There were used three types of meats (turkey meat without bones and skin, lean beef, chicken liver), vegetables (peas, broccoli, and iceberg lettuce), fruits, brown rice and oat bran and Psylium. Premium dry food for cats is of high quality and has a high level of animal protein which makes it appealing to felines. Premium food for dogs with medium level of activity is a complete and balanced food. It uses quality ingredients and due to the percentage of energy from protein concentration and clam extract, helps to prevent overweight and associated risks on bones and joints.

Dry food of veterinary diet type for cats, is a complete food to the sterilised cats that tend fattening because of internal changes produced in the organism. Dry food of veterinary diet type used for the weight control management destined to dogs has also a high content of fibre and low fat as the one for cats. This is a balanced dietetic food for adult dogs having indications in the treatment and prevention of obesity, diabetes, constipation, hyperlipidaemia accompanied by excess weight.

A complete clinical examination was performed, the body score was assessed (there was used a scoring system of 1 to 9 belonging to Royal Canin), as well as the level of activity and the quality of life. There were identified the risk factors related to the environment and individual and it was determined the number of calories needed daily. Monitoring and enforcing of these steps were made at each visit to the patient, from the implementation of the Protocol from 2 in 2 weeks, during which adjustments were made, then monthly and after reaching the ideal weight, during the monitoring period.

\section{RESULTS AND DISCUSSION}

Cooked food given to dogs suffering from obesity had not consistent results, fluctuations in weight being great (Tab. 1.). On the curve of weight loss there was recorded plateau period, while the weight has stagnated, weight loss occurred in a longer period. Due to increased palatability tachyphagia appeared sometimes accompanied by vomiting. Satiety was not long, intestinal transit was increased because of the composition and increased water percentage (D.M. <25\%). The cellulose content of the food was low $(0.8 \%)$ as well as the protein content (13.12\%). Corporal score was improved, as well as quality of life and level of activity, the caloric restriction being 30\% - 40\%. Percentage of weekly slimming had large variations between $0.4 \%$ and $2 \%$, the average being $0.41 \%-0.47 \%$ (Fig.1). The majority of adjustments were done both during the protocol and to the daily caloric intake implicitly to the default amount of daily food and to the activity level. It is recommended a more increased activity level compared to that applied to the administration of premium dry food diet.

Using veterinary diet both in dogs' and cats' food with overweight and obesity had the most consistent results, weight loss being uniform, primarily due to a high content of protein and fibres in the detriment of carbohydrates and fats, the proportion between plastic and energetic elements being very well balanced (Tab. 2., 3.). The high level of $16 \%$ cellulose gives the animal the feeling of satiety, even if metabolizable energy has a lower value compared to the other types of 
Tab. 1. Cooked food - dogs

\begin{tabular}{ccccc}
\hline & Current weight $(\mathrm{kg})$ & 40.34 & Initial activity level score & -1 \\
\cline { 2 - 5 } Case 1 & Ideal weight $(\mathrm{kg})$ & 31.1 & Final activity level score & 0 \\
\cline { 2 - 5 } & Final weight $(\mathrm{kg})$ & 36 & Initial life quality score & -1 \\
\cline { 2 - 5 } & Initial body score & 8 & Final life quality score & +1 \\
\cline { 2 - 5 } & Final body score & 7 & Number of adjustments/protocol & 5 \\
\cline { 2 - 5 } & Kcal/day during the protocol & 660 & Kcal/day recommended & 1100 \\
\cline { 2 - 5 } Kcal/day during maintenance & - & Kcal consumed/ day initially & 1808 \\
\hline Case 2 & Current weight (kg) & 30.56 & Initial activity level score & 0 \\
\cline { 2 - 5 } & Ideal weight (kg) & 27.61 & Final activity level score & +1 \\
\cline { 2 - 5 } & Final weight (kg) & 27.73 & Initial life quality score & +1 \\
\cline { 2 - 5 } & Initial body score & 6 & Final life quality score & 0 \\
\cline { 2 - 5 } & Final body score & $5 / 4$ & Number of adjustments/protocol & 6 \\
\cline { 2 - 5 } & Kcal/day during the protocol & 780 & Kcal/day recommended & 1020 \\
\cline { 2 - 5 } & Kcal/day during maintenance & 1000 & Kcal consumed/ day initially & 1658 \\
\hline
\end{tabular}

Tab. 2. Dry food diet - dogs

\begin{tabular}{|c|c|c|c|c|}
\hline \multirow{7}{*}{ Case 3} & Current weight $(\mathrm{kg})$ & 25.56 & Initial activity level score & -1 \\
\hline & Ideal weight (kg) & 21.3 & Final activity level score & +1 \\
\hline & Final weight $(\mathrm{kg})$ & 21.35 & Initial life quality score & -1 \\
\hline & Initial body score & 7 & Final life quality score & 0 \\
\hline & Final body score & 5 & Number of adjustments/protocol & 3 \\
\hline & Kcal/day during the protocol & 873 & Kcal/day recommended & 970 \\
\hline & Kcal/day during maintenance & 900 & Kcal consumed/ day initially & $1595-1624$ \\
\hline \multirow{7}{*}{ Case 4} & Current weight (kg) & 28.32 & Initial activity level score & 0 \\
\hline & Ideal weight (kg) & 21.78 & Final activity level score & +1 \\
\hline & Final weight $(\mathrm{kg})$ & 21.75 & Initial life quality score & +1 \\
\hline & Initial body score & 8 & Final life quality score & 0 \\
\hline & Final body score & 5 & Number of adjustments/protocol & 3 \\
\hline & Kcal/day during the protocol & 765 & Kcal/day recommended & 850 \\
\hline & Kcal/day during maintenance & 820 & Kcal consumed/ day initially & 1606 \\
\hline
\end{tabular}

diet used. The protein percentage (30\%) higher compared to premium and cooked food, helps to achieve the feeling of satiety and also promotes healthy weight loss without loss of muscle mass.

The percentage of weight loss was between 0.9 to $2 \%$ per week, corresponding to the current trend in the nutritional management of obesity in dogs and cats, with weight loss between 0.70 $1.1 \%$ starting from the weight of the animals at the beginning of the diet and not from the weight of the breed standard. On average throughout the study in cats was recorded a $0.70 \%-0.95 \%$ percentage, and $0.73 \%-1.10 \%$ in dogs (Fig. 1.). This diet did not require periodic quantity adjustments to those recommended by the manufacturer, and the final body score of the patients in the experimental protocol was ideal or close to that ideal.

Premium dry food administered in dogs and cats with overweight and obesity in the nutritional management had inconsistent results (Tab. 4., 5.). During the study, the patients had a fluctuating weight, by gaining and losing weight or stalling. Caloric restriction applied in this protocol was $30-40 \%$, the amount of food being lower than that recommended by the manufacturer. The animal had any feeling of satiety, the food having a low fibre content $(2.5 \%)$ and having a greater caloric 
Tab. 3. Dry food diet - cats

\begin{tabular}{clccc}
\hline & Current weight (kg) & 10.88 & Initial activity level score & -1 \\
\cline { 2 - 5 } Case 5 & Ideal weight (kg) & 7.77 & Final activity level score & 0 \\
\cline { 2 - 5 } & Final weight (kg) & 8.63 & Initial life quality score & -1 \\
\cline { 2 - 5 } & Initial body score & 9 & Final life quality score & 0 \\
\cline { 2 - 5 } & Final body score & 7 & Number of adjustments/protocol & 2 \\
\cline { 2 - 5 } & Kcal/day during the protocol & 342 & Kcal/day recommended & $370-380$ \\
\hline & Kcal/day during maintenance & - & Kcal consumed/ day initially & 1026 \\
\cline { 2 - 5 } Case 6 & 5.15 & Initial activity level score & 0 \\
\cline { 2 - 5 } & Final weight (kg) & 4.29 & Final activity level score & +1 \\
\cline { 2 - 5 } & Initial body score & 7.31 & Initial life quality score & +1 \\
\cline { 2 - 5 } & Final body score & 5 & Fumber of adjustments/protocol & 3 \\
\cline { 2 - 5 } & Kcal/day during the protocol & 225 & Kcal/day recommended & 250 \\
\cline { 2 - 5 } & Kcal/day during maintenance & 250 & Kcal consumed/ day initially & 589 \\
\hline
\end{tabular}

Tab. 4. Premium dry food - dogs

\begin{tabular}{|c|c|c|c|c|}
\hline \multirow{7}{*}{ Case 7} & Current weight (kg) & 28.11 & Initial activity level score & -1 \\
\hline & Ideal weight (kg) & 23.42 & Final activity level score & +1 \\
\hline & Final weight $(\mathrm{kg})$ & 23.5 & Initial life quality score & -1 \\
\hline & Initial body score & 7 & Final life quality score & 0 \\
\hline & Final body score & 5 & Number of adjustments/protocol & 5 \\
\hline & Kcal/day during the protocol & 624 & Kcal/day recommended & 1020 \\
\hline & Kcal/day during maintenance & 800 & Kcal consumed/ day initially & 1420 \\
\hline \multirow{7}{*}{ Case 8} & Current weight $(\mathrm{kg})$ & 38.74 & Initial activity level score & -1 \\
\hline & Ideal weight $(\mathrm{kg})$ & 29.8 & Final activity level score & 0 \\
\hline & Final weight $(\mathrm{kg})$ & 31.60 & Initial life quality score & -1 \\
\hline & Initial body score & 8 & Final life quality score & 0 \\
\hline & Final body score & $7 / 6$ & Number of adjustments/protocol & 5 \\
\hline & Kcal/day during the protocol & 734 & Kcal/day recommended & 1050 \\
\hline & Kcal/day during maintenance & - & Kcal consumed/ day initially & 1360 \\
\hline
\end{tabular}

density. Because of the moderate protein quantity (21.5\%) during the weight loss process, it was also lost muscle mass. This aspect influenced as well lack of satiety sensation of the animals. The percentage of weight loss was averaged in cats at $0.73 \%-0.82 \%$ and in dogs at $0.20 \%-0.35 \%$, the weekly percentage was not constant and varied between $0.5 \%$ and $2.6 \%$ in dogs and $0.3 \%-1 \%$ in cats (Fig. 1.). The body score was improved being ideal or near ideal. There have been done many adjustments related to the activity level and to the quantity of food given during the protocol. Quality of life and level of activity score improved.
Impact of body weight in dogs was best illustrated in a study made by Kealy et al. in 2012, a study that lasted 14 years. The clinical study was conducted on a group of Labrador retriever breed who were fed $25 \%$ less than the data indicated by the manufacturer, and another control group consisting of the same race. The group fed with a greater amount of food presented cases of overweight and weighed on average 26\% more than the group fed with a smaller amount of food. The group fed with a smaller amount had a score of 4-5 (ideally) from a numbering system of 9 . The difference in weight was significant and influenced longevity, the less fed group lived on average 13 
Tab. 5. Premium dry food - cats

\begin{tabular}{ccccc}
\hline & Current weight $(\mathrm{kg})$ & 3.5 & Initial activity level score & -1 \\
\cline { 2 - 5 } Case 9 & 3.18 & Final activity level score & 0 \\
\cline { 2 - 5 } & Final weight $(\mathrm{kg})$ & 3.22 & Initial life quality score & 0 \\
\cline { 2 - 5 } & Initial body score & 6 & Final life quality score & +1 \\
\cline { 2 - 5 } & Final body score & 5 & Number of adjustments/protocol & 4 \\
\cline { 2 - 5 } & Kcal/day during the protocol & 137 & Kcal/day recommended & 210 \\
\cline { 2 - 5 } Kcal/day during maintenance & 200 & Kcal consumed/ day initially & 512.2 \\
\hline Carrent weight $(\mathrm{kg})$ & 5.18 & Initial activity level score & -1 \\
\cline { 2 - 5 } & Ideal weight $(\mathrm{kg})$ & 4.31 & Final activity level score & 0 \\
\cline { 2 - 5 } & Final weight $(\mathrm{kg})$ & 4.93 & Initial life quality score & 0 \\
\cline { 2 - 5 } & Initial body score & 6 & Final life quality score & 0 \\
\cline { 2 - 5 } & Final body score & 6 & Number of adjustments/protocol & 4 \\
\cline { 2 - 5 } & Kcal/day during the protocol & 175 & Kcal/day recommended & 250 \\
\cline { 2 - 5 } & Kcal/day during maintenance & - & Kcal consumed/ day initially & 565 \\
\hline
\end{tabular}

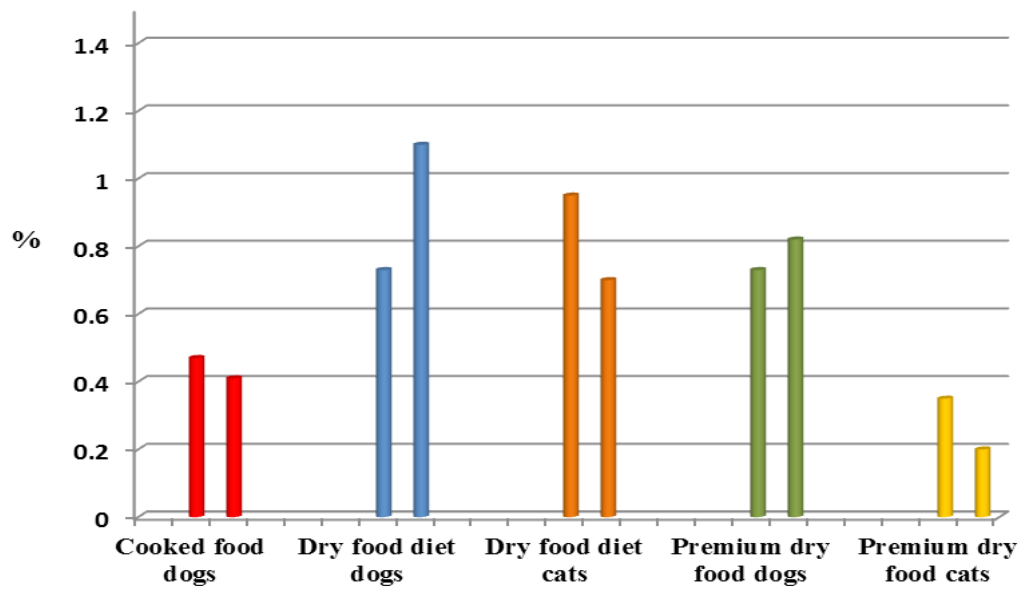

Fig. 1. Average weekly percentage of weight loss

years, and the on fed more lived less than 12 years. Medical problems in the musculoskeletal system appeared earlier with 2.1-3 years in the control group.

A study conducted in 2014 by Brooks et al., reveals that for a constantly and healthy weight loss it has to be associated with caloric restriction depending on selected diet, level of activity, type of exercise and other factors that lead to beneficial changes to patient and customer behaviour. Caloric reduction may be between 10\% - 20\% and $60 \%$ of the daily recommended calories for an ideal body weight. Quantities may differ from those recommended by the manufacturer.

There are controversial data about the use of the fibre and the appearance of satiety effect. Some studies suggest that a diet containing a percentage of $12-16 \%$ of the dry substance in the composition would not have the desired effect. Other studies have shown that suppression of appetite and satiety apparition came when given a food containing 21\% fibre. Diet had effect after caloric restriction and adjustment and improvement of the activity level. (German, 2006a; German, 2006b).

\section{CONCLUSION}

Following the results obtained at protocol completion, we can say that the best results were achieved in managing dry food, being more recommended in case of overweight and obesity in dogs and cats than cooked food. Comparing the dry food and the premium diet, the best results have been observed in case of diet administration. 
We recommend the administration of dry food in overweight and obesity problems in dogs and cats with the mention that without subsequently adjusting the level of activity, no obvious result appear, without re-weight gain effect.

The recommended amount of food must be weighed daily, and food diet should be associated with adjusting the level of activity and exercise whenever needed. Once the animal has reached his ideal weight, it should continue to have a moderate activity level. Whatever diet applied, the animal should be weighed frequently and must be kept records of the percentage of weakening percentage which should not exceed 1-2\% weekly. Adjustments are made on the amount of food, respectively to the daily intake of calories, whenever needed. Although the satiety effect occurs when the animals reach their ideal weight, the Rebound effect was not present.

\section{REFERENCES}

1. Brooks D, Churchill J, Fein K, Linder D, Linder D, Michel K, Tudor K, Ward E, Witzel A (2014). Weight Management Guidelines for Dogs and Cats. Veterinary practice guidelines. JAAHA.ORG, 1-11.

2. Brooks D, Churchill J, Fein K, Linder D, Zoran D (2010). Obesity in Dogs and Cats: A Metabolic and Endocrine Disorder. Review Article: Veterinary Clinics of North America: Small Animal Practice, Volume 40, 221-239.
3. Burns K. (2013). Why is Rocky so Stocky. Obesity is a Disease! Journal of the North American Veterinary Technician Association. Convention, 16-19.

4. Burns KM, Towell TL (2011). Owner Education and Adherence. In Towell TL. Practical Weight Management in Dogs and Cats. Ames, IA: Wiley-Blackwell, 3-21.

5. Fascetti J, Delaney F (2012). Applied Veterinary Clinical Nutrition. Wiley-Black well, 3-125.

6. German AJ (2006a). The growing problem of obesity in dogs and cats. J Nutr. 136 (7): 1940-1946.

7. German AJ (2006b). Clinical risks associated with Obesity in companion animals. Waltham, vol.16:1,1-4.

8. German AJ, Holden SL, Mason SL (2011). Imprecision when using measuring cups to weigh out extruded dry kibbled food. J Anim, Physiol Anim Nutr (Berl);95(3):36873.

9. German AJ, Holden SL, Wiseman-Orr ML, Reid J, Nolan AM, Biourge V, Morris PJ, Scott EM (2012). Quality of life is reduced in obese dogs but improves after successful weight loss. Volume 192, Issue 3, , Pages 428-434.

10. Kealy RD, Lawler DF, Ballam JM, Mantz SL, Biery DN, Greeley EH, Lust G, Segre M, Smith GK, Stowe HD (2002). Effects of diet restriction on life span and age-related changes in dogs. Journal of the American Veterinary Medical Association 220, 1315-1320.

11. Linder DE, Parker VJ (2016). Dietary Aspects of Weight Management in Cats and Dogs. Veterinary Clinics of North America: Small Animal Practice Volume 46, Issue 5, Pages 869-882.

12. Pibot P, Biourge V, Elliot D (2010). Encyclopedia of Canine Clinical Nutrition, 3-61. 